\title{
Distinctive Spectroscopic Characteristics Observed in Colloidal Perovskite CsPbBr 3 Quantum Dot
}

Received 15 July, 2021; revised 19 July, 2021; accepted 29 July, 2021

\author{
Sangeun Cho, Sam Kyu Noh, Hyungsang Kim, and Hyunsik Im* \\ Division of Physics and Semiconductor Science, Dongguk University, Seoul 04620, Republic of Korea
}

*Corresponding author E-mail: hyunsik7@dongguk.edu

\begin{abstract}
Distinctive spectroscopic characteristics are observed in colloidal perovskite $\mathrm{CsPbr}_{3}$ quantum dot (QD) dispersed in octane doped with PPO. As the content of $\mathrm{CsPBr}_{3}-\mathrm{QD}$ increases, the emission and the absorption energies decrease due to QD-cluster formed in case of the inter-QD distance smaller than diameter of QD. Spectroscopic evidences supporting QD-cluster are found in the Urbach energy and the carrier lifetime. Unusual absorption lines showing explicit QD dependence are observed in Fourier-transform infrared spectra, which is explained by vibrational modes of bound $\mathrm{PPO}$ hybridized with $\mathrm{CsPbBr} 3-\mathrm{QD}$.
\end{abstract}

Keywords: Perovskite, $\mathrm{CsPbB}_{3}$, Quantum dot, Time-resolved photoluminescence, Absorption, Fourier-transform infrared spectroscopy

\section{Introduction}

By virtue of distinguished characteristics in light emission and absorption, the perovskite ( $\mathrm{Px})$ structure has abruptly emerged as a new semiconductor system [1]. The application has been widely spreading over all fields of optoelectronic devices, such as solar cells [2,3], lightemitting devices [4], photodetectors [5], $\mathrm{X} / \boldsymbol{\gamma}$-ray detectors [6,7], and $\mathrm{THz}$ emitters [8]. In these, the solar cell has rapidly developed in 10 years since Miyasaka [9] first reported the efficiency of $3 \%$ in 2009. By introduction of organic-inorganic hybrid $\mathrm{Px}\left(\mathrm{FAPbI}_{3} / \mathrm{MAPbBr}_{3}\right)$ and of pseudo-halide anion formate $\left(\mathrm{HCOO}^{-}\right)$, the efficiency jumps up to 24.23 (2019) [2] and $25.6 \%$ (2021) [3], respectively, whose values are superior to that of Si-based solar cell. In particular, all-inorganic $\mathrm{CsPbX}_{3}(\mathrm{X}=\mathrm{Cl}, \mathrm{Br}, \mathrm{I})$ is drawing attention as new Px candidate that could replace the hybrid Px with drawback like phase decomposition. Nano-crystalline $\mathrm{CsPbX}_{3}$ exhibits quantum-dot (QD) behavior $[10,11]$ that shows extremely high photoluminescence quantum yield (PLQY) and photoelectric conversion efficiency (PCE). In addition, it has been known that the wavelength modulation is available in the whole range of visible light $(\sim 430-780 \mathrm{~nm})$ by simply substituting halogen element $(\mathrm{X})$. Therefore, a variety of Px-based materials and their photoelectric applications are now under study [10-13].

Figure 1 illustrates some experimental results obtained in previous study [14]. The specimens used were $\mathrm{CsPbX}_{3}$-QDs dispersed in solvent (octane, $\mathrm{C}_{8} \mathrm{H}_{18}$ ) with a small amount of fluorescent substance of PPO (2,5-diphenyloxazole, $\mathrm{C}_{15} \mathrm{H}_{11} \mathrm{NO}$ ), which was successfully applied to high-sensitivity liquid X-ray scintillator. Figure 1(a) is optical photographs for representative 5 samples synthesized in our laboratory, Figs. 1(b)/1(c) the light emission of each sample under irradiation of UV (365 nm)/X-ray (6 MV), and Figs. 1(d)/1(e) representative photoluminescence/radioluminescence (PL/RL) spectra. Figure 1(f) is a demonstrative $\mathrm{X}$-ray image taken by the $\mathrm{CsPbBr}_{3}-\mathrm{QD}$ scintillator. Figures $1(\mathrm{~d}) / 1(\mathrm{e})$ reveal that each $\mathrm{CsPbX}_{3}$-QD sample of different component/composition (X) has a unique spectrum, and added $\mathrm{PPO}$
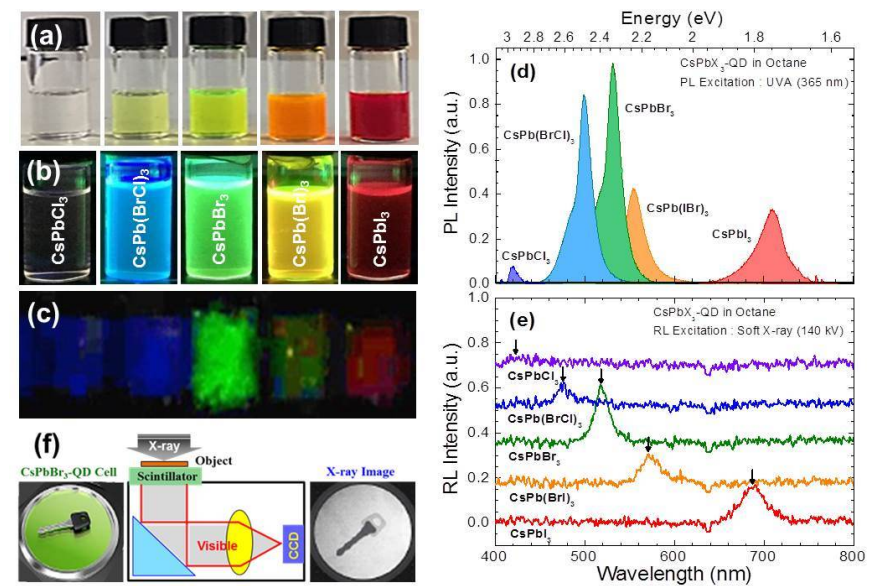

Figure 1. Photographs for emission of colloidal $\mathrm{CsPbX} 3-\mathrm{QD}$ samples taken under (a) white light, (b) UV (365 nm), and (c) X-ray (6 MV). (d) PL spectra, (e) RL spectra, and $(\mathrm{f})$ schematic diagram of $\mathrm{X}$-ray imaging system and $\mathrm{X}$-ray image obtained by using lab-fabricated $\mathrm{CSPbBr}_{3}-\mathrm{QD}$ liquid scintillator.

does not perturb spectral characteristics of QD in the visible light region at all. The details on the lab-fabricated $\mathrm{PPO}$-assisted $\mathrm{Cs} \mathrm{PbBr}_{3}-$ QD scintillator are described in previous report [14].

In this study, we report some unusual spectroscopic behaviors observed in colloidal $\mathrm{CsPbBr}_{3}$-QD doped with PPO. The formation of QD-cluster is proposed on the basis of PL and absorption (AB) spectra showing $\mathrm{QD}$ dependence. The Urbach energy manipulated from $\mathrm{AB}$ spectra and the carrier lifetime identified by time-resolved photoluminescence (TRPL) spectra are discussed as additional data supporting QD-cluster. In Fourier-transform infrared (FTIR) spectroscopy, the 


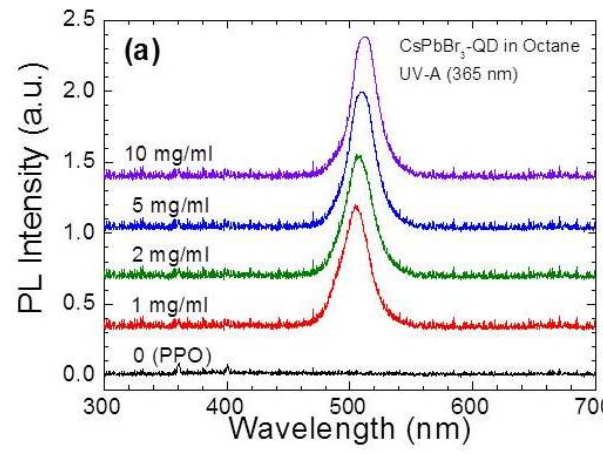

Inter-QD Distance $(\mathrm{nm})$

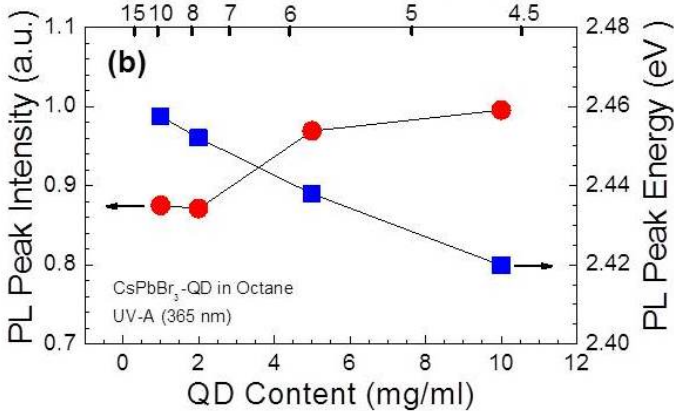

Figure 2. (a) PL spectra and (b) PL peak intensity and energy plotted as a function of QD content.

QD-PPO hybridization is proposed for explanation of distinctive behavior on $\mathrm{AB}$ lines growing with the $\mathrm{QD}$ content.

\section{Experimental details}

$\mathrm{CsPbBr}_{3}$-QD samples used in this study were prepared by the hotinjection synthesis that was introduced by Schmidt et al. [15] and Protesescu et al. [4] for metal-calcogenide QD and metal-hallide Px$\mathrm{QD}$, respectively. First, for Cs-precursor, cesium-carbonate $\left(\mathrm{Cs}_{2} \mathrm{CO}_{3}\right)$ and oleic-acid $(\mathrm{OA})$ were mixed with octadecene (ODE) and reacted by heating in nitrogen atmosphere. $\mathrm{PbBr}_{2}$ precursor was prepared by mixing $\mathrm{PbBr}_{2} / \mathrm{ODE}$ with ligands of $\mathrm{OA}+\mathrm{OLA}$ (oleylamine). When $\mathrm{PbBr}_{2}$ was dissolved, the temperature was raised up to $180{ }^{\circ} \mathrm{C}$ and Cs-precursor was injected. The mixture was quenched in a cooling reservoir and separated $\mathrm{CsPbBr}_{3}-\mathrm{QD}$ powder through centrifugation.

Several tens of milligrams of QD powder were synthesized in a single batch, and a series of $\mathrm{Cs} \mathrm{PbBr}_{3}-\mathrm{QD}$ samples for each spectroscopy were prepared by dispersing QD powder in octane just before measurement. It was confirmed that $\mathrm{CsPbBr}_{3}$-QD samples used in this study exhibited excellent PLQY and unique PL spectra, as demonstrated in Fig. 1.

\section{Results and discussion}

\subsection{Red shift in photoluminescence spectra}

Figure 2 presents (a) PL spectra and (b) a pair of curves for the intensity and the energy of PL peaks of $\mathrm{CsPbBr}_{3}$-QDs plotted as a function of QD content. As the QD content increases from $1 \rightarrow 10$ $\mathrm{mg} / \mathrm{ml}$, the intensity monotonically increases, but the energy abruptly decreases from 2.458 to $2.420 \mathrm{eV}$. It can be easily understood for increase of PL intensity in terms of the number of QDs, but a plausible explanation is required for the anomaly of PL energy.

We have introduced the inter-QD distance as a parameter alternative to the QD content, as denoted on the upper axis of Fig. 2. When the distance between QDs becomes smaller than diameter of QDs, a

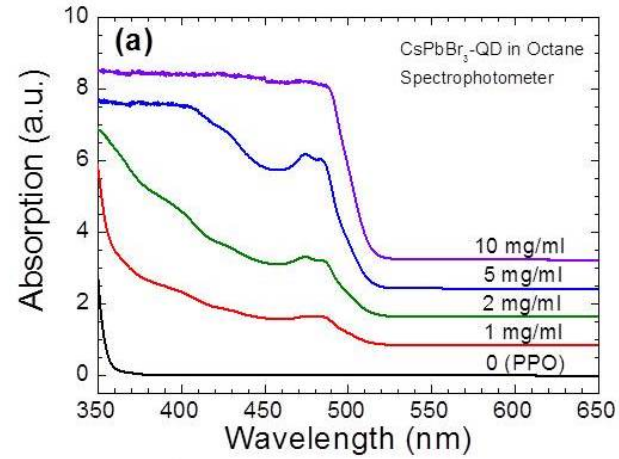

Inter-QD Distance $(\mathrm{nm})$

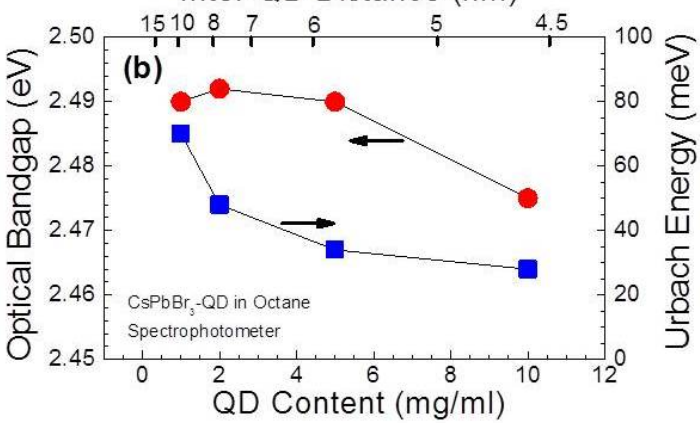

Figure 3. (a) AB spectra and (b) optical bandgap and Urbach energy plotted as a function of QD content.

bulk of QDs adhere and entangle each other to form QD-cluster. The sublevel energy of QD-cluster suffers the red shift that is lower than that of individual QD due to the quantum confinement effect [16]. The QD content increases more, the size of QD-cluster becomes bigger, and the red shift becomes larger.

The average inter-QD distance D introduced to justify the red shift of PL energy was simply manipulated by the equation of

$$
D=\left[M / N_{\mathrm{A}} d\right]^{1 / 3},
$$

where $N_{\mathrm{A}}$ is the Avogadro number $\left(6.02 \times 10^{23} \mathrm{~mol}^{-1}\right)$, and $M$ and $d$ the molecular weight of $\mathrm{CsPbBr}_{3}$ and the $\mathrm{QD}$ content, respectively. As the QD content $(d)$ changes from $1 \rightarrow 10 \mathrm{mg} / \mathrm{ml}$, the inter-QD distance $(D)$ approaches from 9.8 to $4.6 \mathrm{~nm}$ whose values are comparable to or smaller than QD diameter of 8-12 $\mathrm{nm}$. These figures confirm that the inter-QD distance is sufficiently close to form QD-clusters.

\subsection{Optical bandgap and Urbach energy in absorp- tion spectra}

Experimental results supporting the QD-cluster formation can be seen in the $A B$ spectra. Figure 3 shows (a) $A B$ spectra and (b) a pair of plots for the optical bandgap $\left[E_{\mathrm{g}}(O)\right]$ and the Urbach energy $[E(U)]$ of $\mathrm{CsPBBr}_{3}$-QD.

$E_{\mathrm{g}}(O)$ was determined from the intercept of the relationship known to Tauc plot as

$$
(\alpha E)^{n}=k\left[E-E_{\mathrm{g}}(O)\right],
$$

where $\alpha$ is $\mathrm{AB}$ coefficient, $n=2$ for the direct allowed transition, and $k$ a proportional constant. As the QD content increases from $1 \rightarrow 10$ $\mathrm{mg} / \mathrm{ml}, E_{\mathrm{g}}(O)$ decreases from 2.490 to $2.475 \mathrm{eV}$ whose values are higher than the PL peak energy by $32-55 \mathrm{meV}$ equivalent to $6-12 \mathrm{~nm}$ in wavelength. The difference is well known to the Stokes shift that is frequently observed in normal spectroscopy, and decreasing $E_{\mathrm{g}}(O)$ may be the other evidence for the QD-cluster formation. 


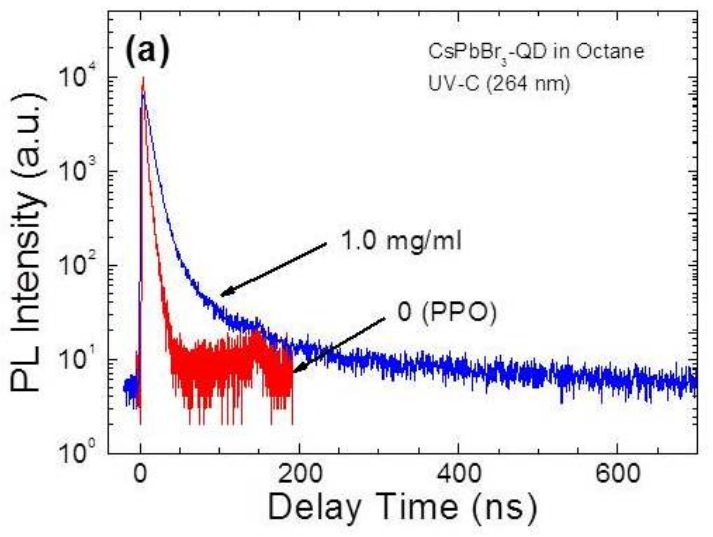

Inter-QD Distance $(\mathrm{nm})$

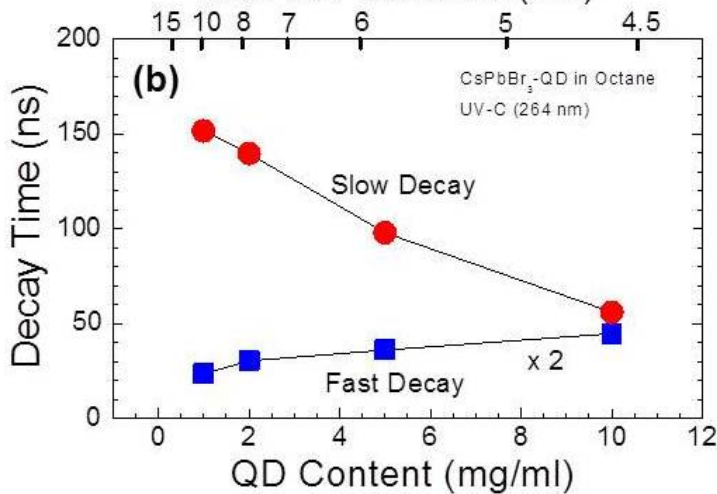

Figure 4. (a) TRPL spectra and (b) carrier lifetimes for fast and slow decays plotted as a function of QD content.

The Urbach energy $E(U)$ was estimated from the exponential term expressing the spectral abruptness near $\mathrm{AB}$ edge as

$$
\alpha=\alpha_{0} \exp [E / E(U)]
$$

where $\alpha_{0}$ is the value at $E=0 . E(U)$ is a parameter corresponding to the linewidth energy of band-tail formed by defects. As shown in Fig. 3, $E(U)$ monotonically decreases with increasing QD content, which indicates surface defects reduced by the QD-cluster formation. In other words, the surface-to-volume ratio (SVR) of QD-cluster formed by a bulk of QDs is smaller than those of individual QDs. Therefore, the surface trap due to dangling bonds becomes decreased, which leads to reduction of $E(U)$.

\subsection{Carrier lifetime in time-resolved photolumines- cence spectra}

We suggest the carrier lifetime as an additional result for QD-cluster. Figure 4 presents (a) TRPL spectra and (b) a couple of plots for the fast/slow decay time taken at PL peaks for $\mathrm{CsPbBr}_{3}$-QDs, which was analyzed by double-exponential decay function. As the QD content increases, the fast decay exhibits a minor increase in the range of 10-20 $\mathrm{ns}$, but the slow decay experiences a rapid decrease from 150 to $50 \mathrm{~ns}$.

Table I. Summary of 6 AB lines observed in FTIR spectra and suggested origin.

\begin{tabular}{llll}
\hline AB Line & QD Dependence & Mode & Suggested Origin \\
$\mathbf{1 4 6 5} \mathbf{c m}^{-1}$ & None & C-H Bending & Individual PPO \\
$\mathbf{1 3 7 8} \mathbf{~ c m}^{-1}$ & Weak & C-H Bending & Hybrid QD-PPO \\
$\mathbf{1 2 4 0} \mathbf{~ c m}^{-1}$ & Weak & C-N Stretching & Hybrid QD-PPO \\
$\mathbf{1 0 5 6} \mathbf{c m}^{-1}$ & Strong & C-O Stretching & Hybrid QD-PPO \\
$\mathbf{8 8 7} \mathbf{c m}^{-1}$ & Weak & C=C Bending & Hybrid QD-PPO \\
$\mathbf{7 1 9} \mathbf{c m}^{-1}$ & None & C=C Bending & Individual PPO \\
\hline
\end{tabular}

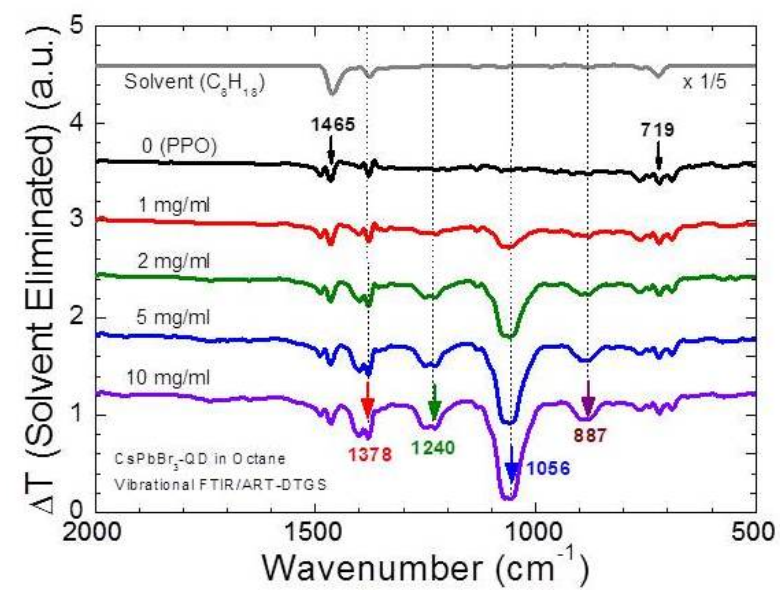

Figure 5. FTIR spectra of solvent $\left(\mathrm{C}_{8} \mathrm{H}_{18}\right)$ and colloidal $\mathrm{CSPbBr}_{3}-\mathrm{QDS}$ with different QD content.

The fast decay reflects an average lifetime for carriers captured at surface traps such as dangling bonds. As the size of QD-cluster grows larger, the number of surface defects reduces and the average lifetime becomes longer. On the other hand, the slow decay time includes an information on the recombination rate $(R)$ of free electron/hole $(n / p)$ in conduction/valence sublevel. As previously discussed in PL/AB spectra, the red shift of the QD sublevel due to the cluster formation results in enhancement of the recombination rate $(R \sim n p)$, which makes the carrier lifetime short.

\subsection{Bonding modes in Fourier-transform infrared spectra}

An interesting behavior was observed in infrared spectroscopy. Fig ure 5 shows FTIR spectra of colloidal $\mathrm{CsPbBr}_{3}-\mathrm{QDs}$ that are basically the same as samples used in this study. It is noted that the QD spectrum displays the net intensity eliminated by the solvent background (topmost) from the raw QD signal via a graphic software. Six AB lines were found in FTIR spectra, and they could be categorized into 2 groups. One is Group-A for 2 outermost AB lines $\left(1465,719 \mathrm{~cm}^{-1}\right)$ with invariable intensity over all QD spectra, and the other is Group-B that consists of 4 inner $A B$ lines $\left(1378,1240,1056,887 \mathrm{~cm}^{-1}\right)$ showing explicit QD dependence.

Table I is a summary on $6 \mathrm{AB}$ lines observed in FTIR spectra. The bonding modes suggested were retrieved from the Reference Data Book [17]. Considering that PPO spectrum is composed of only 2 GroupA lines invariable for all $\mathrm{QD}$ spectra, it is clear that the $2 \mathrm{AB}$ lines $\left(1465 / 719 \mathrm{~cm}^{-1}\right)$ are attributed to $\mathrm{C}-\mathrm{H} / \mathrm{C}=\mathrm{C}$ bending of individual PPO molecules $\left(\mathrm{C}_{15} \mathrm{H}_{11} \mathrm{NO}\right)$.

Taking consideration of spectral evolution of Group-B growing with the QD content, Group-B should be strongly correlated with QDs, absolutely opposite to Group-A. Here is an ambiguity in GroupB. Despite that Group-B exhibits explicit dependence on $\mathrm{CsPbr}_{3}-$ $\mathrm{QD}$, no vibrational mode related with $\mathrm{Cs}-\mathrm{Pb}-\mathrm{Br}$ bonds exists in the whole FTIR spectral range of $4000-400 \mathrm{~cm}^{-1}$. The contradiction of Group-B can be explained by hybridization of QD $\left(\mathrm{CsPbr}_{3}\right)$ and $\mathrm{PPO}$ $\left(\mathrm{C}_{15} \mathrm{H}_{11} \mathrm{NO}\right)$. The hybridized system was already proved both theoretically and experimentally in previous study [14], and the schematic model of PPO hybridized with QD is sketched in Fig. 6. In this configuration, negatively charged $\mathrm{N}$ of $\mathrm{PPO}$ and positively charged $\mathrm{Pb}$ of $\mathrm{QD}$ form ionic bond, so PPO molecule is tightly bound to $\mathrm{CsPbr}_{3}-\mathrm{QD}$ via $\mathrm{Pb}-\mathrm{N}$ bond. Here, we can suggest exceptional vibrational modes attributed to oxazole radical $\left(\mathrm{C}_{3} \mathrm{HNO}=\right)$ in PPO bound to heavy QDmolecule/QD-cluster. Four kinds of vibrational modes of C-H, C-N, $\mathrm{C}-\mathrm{O}$, and $\mathrm{C}=\mathrm{C}$ are available in $\mathrm{C}_{3} \mathrm{HNO}=$, and each mode can be corre- 


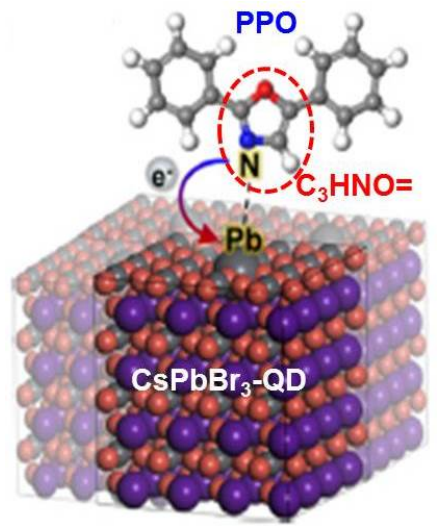

Figure 6. Schematic picture of hybridized QD-PPO $\left(\mathrm{CsPbBr}_{3}-\mathrm{C}_{15} \mathrm{H}_{11} \mathrm{NO}\right)$. (This figure is the duplicate of Fig. 2(e) in Ref [14].)

sponded to one of 4 Group-B lines, as listed in Table I. The AB intensity of C-O stretching mode stronger than those of the other modes $(\mathrm{C}-\mathrm{H}$, $\mathrm{C}-\mathrm{N}, \mathrm{C}=\mathrm{C}$ ) may be due to difference in strength of dipole moment.

\section{Conclusions}

We discussed some unusual spectroscopic characteristics of colloidal $\mathrm{CsPbBr}_{3}$-QDs observed in PL, AB, TRPL, and FTIR spectra. The anomaly of PL and $\mathrm{AB}$ energies decreased with increase of $\mathrm{QD}$ content was explained by QD-cluster formed by a bulk of QDs. The Urbach energy $[E(U)]$ of $A B$ spectra and the carrier lifetime of TRPL spectra were suggested as additional data supporting the QD-cluster formation.

The FTIR spectra exhibited 2 kinds of distinctive characteristics. The former was $2 \mathrm{AB}$ lines (Group-A; 1465, $719 \mathrm{~cm}^{-1}$ ) with invariable intensity over all QD spectra that were due to bending modes of $\mathrm{C}-\mathrm{H} / \mathrm{C}=\mathrm{C}$ of individual $\mathrm{PPO}$, and the latter was $4 \mathrm{AB}$ lines (GroupB; 1378, 1240, 1056, $887 \mathrm{~cm}^{-1}$ ) with explicit QD dependence that were attributed to bending/stretching modes of $\mathrm{C}-\mathrm{H} / \mathrm{C}-\mathrm{N} / \mathrm{C}-\mathrm{O} / \mathrm{C}=\mathrm{C}$ of bound PPO hybridized with QD-molecule/cluster. Though the origin of QD-dependent Group-B lines was identified as PPO bound to $\mathrm{QD}$, we judge that the observation of QD-dependent FTIR lines is a very distinctive result.

\section{Acknowledgements}

This work was supported by the National Research Foundation (NRF) of Korea (Grant No. 2021R1F1A1062672, 2019R1I1A1A01048852) and Technology Innovation Program (Grant No. 20013597) funded by the Ministry of Trade, Industry \& Energy.

\section{References}

[1] Y. Kanemitsu and T. Handa, Jpn. J. Appl. Phys. 57, 090101 (2018).

[2] E. H. Jung, N. J. Jeon, E. Y. Park, C. S. Moon, T. J. Shin, T. Y. Yang, J. H. Noh, and J. Seo, Nature 567, 511 (2019).

[3] J. Jeong et al., Nature 592, 381 (2021).

[4] L. Protesescu, S. Yakunin, M. I. Bodnarchuk, F. Krieg, R. Caputo, C. H. Hendon, R. X. Yang, A. Walsh, and M. V. Kovalenko, Nano Lett. 15, 3692 (2015).

[5] M. V. Kovalenko, L. Protesescu, and M. I. Bodnarchuk, Science 358, 745 (2017).

[6] Y. C. Kim, K. H. Kim, D. Y. Son, D. N. Jeong, J. Y. Seo, Y. S. Choi, I. T. Han, S. Y. Lee, and N. G. Park, Nature 550, 87 (2017).

[7] M. Gandini et al., Nat. Nanotechnol. 15, 462 (2020).
[8] B. Guzelturk et al., Adv. Mater. 30, 1704737 (2018).

[9] A. Kojima, K. Teshima, Y. Shirai, and T. Miyasaka, J. Am. Chem. Soc. 131, 6050 (2009).

[10] A. R. Kirmani, J. M. Luther, M. Abolhasani, and A. Amassian, ACS Energy Lett. 5, 3069 (2020).

[11] I. W. Cho and M. Y. Ryu, Appl. Sci. Converg. Technol. 29, 28 (2020).

[12] Y. Yun, J. Y. Cho, J. Heo, and S. Lee, Appl. Sci. Converg. Technol. 27, 169 (2018).

[13] J. Oh, W. Y. Jeong, S. Y. Lee, B. Lee, and M. Y. Ryu, Appl. Sci. Converg. Technol. 29, 19 (2020).

[14] S. Cho et al., Light Sci. Appl. 9, 156 (2020).

[15] L. C. Schmidt, A. Pertegás, S. González-Carrero, O. Malinkiewicz, S. Agouram, G. M. Espallargas, H. J. Bolink, R. E. Galian, and J. Pérez-Prieto, J. Am. Chem. Soc. 136, 850 (2014).

[16] J. O. Kim, S. J. Lee, S. K. Noh, Y. H. Ryu, S. M. Choi, and J. W. Choe, J. Korean Phys. Soc. 47, 838 (2005).

[17] IR Spectrum Table \& Chart, Open-access source by SigmaAldrich Product, Merck KGaA (2021). 